\title{
Design of Wireless Swallowable Capsule with Minimum Power Consumption and High Resolution Images
}

\author{
I. G. Intzes* and H. Meng \\ Department of Electronic and Computer Engineering, Brunel University London, UK
}

Received 30 June 2015; Accepted 15 September 2016

\begin{abstract}
Medical technology has undergone major breakthroughs in recent years, especially in the area of the examination tools for diagnostic purposes. The traditional examination method for the diagnosis of gastrointestinal diseases is gastroscopy with the use of an endoscope. Wireless camera pill has created a new perspective for engineers and physicians. After years of great innovation, commercial swallowable pills have been produced and applied in clinical practice. These pills can cover the examination of the gastrointestinal system and provide to the physicians not only a lot more useful data that is not available from the traditional methods, but also elimination of the use of the painful endoscopy procedure. In this paper, a new design of the wireless swallowable pills has been proposed. It takes advantage of the benefits of every sub-system, like camera lenses, image compressor and RF sub-system. In this way our system can provide enough and accurate data to the physicians.
\end{abstract}

Keywords: FPGA, ASIC, VLSI, JPEG2000, wavelet, OOK, WCE, smartpill, capsule endoscopy, modulation.

\section{Introduction}

Wireless capsule endoscopy (WCE) is a state of art technology for medical diagnoses of gastrointestinal diseases and illnesses. The idea was originally started in 1950 when a lot of research effort had been made to improve the diagnoses abilities using new technologies. Through the years, WCE has been named by different names like, smartpill, wireless endoscopy, video capsule, etc. The very first attempts of WCEs, use transmitters with low frequency carrier and the diagnosis was based only from sensor's data like temperature, $\mathrm{pH}$, and pressure. Due to the technology evolution, WCEs were used for the diagnosis by small cameras with low resolution, combining sensors' data. The final diagnosis was more accurate.

The most common diseases that WCEs can diagnose are some types of cancer, Crohn's disease and obscure gastrointestinal bleeding. By the use of WCE, tissue can be taken from specific areas for biopsy.

For medical practice, capsule endoscopy was, introduced commercially by the Israeli company GivenImaging, Inc. [1] at 2009. The first capsules were designed for investigating the small bowel because it was hard to investigate with the traditional techniques. These commercial products were able to provide low resolution images at low frame rate. Nowadays there are capsules dedicated for specific investigation areas, such as esophagus (PillCam ESO) [1] and colon (PillCam COLON) [1].

Apart from GivenImaging, there are other companies offering similar products. The most recent product of GivenImaging, contains two cameras, (PillCam COLON) [1] horizontally placed in the pill, one in the front of the pill and one in the back, and it can support up to 30 frames per

*E-mail address: intzes.ioannis@gmail.com

ISSN: 1791-2377 @ 2016 Eastern Macedonia and Thrace Institute of Technology. All rights reserved. second. The academic/scientific community has also taken part in the evolution of this technology and has come up with interesting products and ideas.

One of the most popular ones is the camera capsule. The evolution of technology and the tiny size of cameras and microprocessors gain the interests of the scientific community to design and improve the existing systems to the next level.

In this paper we describe the design and implementation of a new system of WCE with low power consumption and high resolution images.

\section{Design of the wireless swallowable capsule}

The endoscopic capsules play a major role in the diagnosis of the gastrointestinal track (GI). The traditional methods are painful for patients and is not possible to examine the entire GI. It is obvious that to achieve the high demands of medical diagnosis of the entire gastrointestinal track, state of art technology must be used.

A wireless capsule, despite its size, consists of a lot of electronic parts. There are sensors, the processing unit, the wireless transmission unit and the power management unit, which is usually integrated with the processing unit.

In the part of the sensors, various types are used like, pressure sensor, temperature sensor [2], inertial sensor, $\mathrm{pH}$ sensor, conductivity sensor and oxygenation sensor. For example, with a $\mathrm{pH}$ sensor the detection of acidic reflux can be diagnosed, or with the electrical conductivity sensor the detection of non-acidic reflux can be diagnosed. [3] Both of these measurements can provide more information, for the final diagnoses, to the physicians.

\subsection{High resolution camera with special lenses}

In our system special lenses are going to be used to produce 360-degree view images. Catadioptric omnidirectional [4] 
lenses are going to be used. The use of such lenses has advantages and disadvantages.

This type of lenses give us more advantages such us the high resolution image capture. This is achievable because high frame rate images are not needed, a few images per second is enough. The disadvantage is the distortion produced by these lenses in the edge of the image but with the use of computer software we can solve this problem.

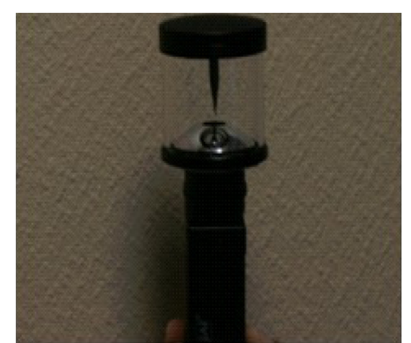

(a)

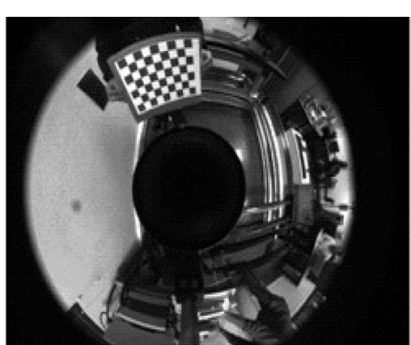

(b)
Fig. 1 Implementation of omnidirectional video cameras by using paraboloidal mirrors. [4]

By this way we can increase the resolution of the captured image. Also the use of these lenses gives us the opportunity to reproduce in a PC (Personal Computer) in $3 \mathrm{D}$, the patients' intestine. Only one camera sensor is needed and that makes the proposed system more energy efficient.

\subsection{Processing Unit}

The design of the Process Unit is implemented on a FPGA development board [5], with a Xilinx SPARTAN-6 FPGA. This part is controlling the whole system. It consists of the below sub-systems.

- Camera control sub-system.

- Sensor control sub-system.

- Image processing sub-system.

- Energy handling sub-system and

- Wireless transmitter.

In the following, the image processing and the wireless transmitter sub-system will be described in details.

The image processing unit uses discrete wavelet transform (DWT) and Huffman encoding for image compression. Especially, for DWT we use a spatial combinative lifting algorithm (SCLA) [6] scheme with 5/3 reversible filter. By this way we reduce by $50 \%$ the multiplications needed and so the computation effort, compared with conventional lifting-based implementation (LBI). In Figure 2 we can see in diagram how the SCLA algorithm is working.

This algorithm is identical to work with real-time pixel data. It takes serially the pixels produced from the camera and outputs the processed image. The proposed algorithm reads serially the image data and produces the $\mathrm{HL}, \mathrm{LH}, \mathrm{HH}$ wavelet coefficients directly of each level decomposition. Simultaneously, the LL coefficients are prepared for the next decomposition level. In our design we implement a 5-level DWT decomposition.

SCLA algorithm is based on the LBI architecture. Due to this feature the memory organization is almost the same. The access to a memory organization like LBI is not sufficient and needs sophisticated computations. The memory organization of the SCLA algorithm consists of four line buffers memories in total. Two line buffers named Line0Line1 for storage of intermediate data of the level decomposition and two buffer memories split in four buffers (half line capacity each), Line2_Leve14, Line3_Level4 and
Line2_LL, Line3_LL. Line2_Level4, Line3_Level4 are used for buffering the raw image data and the last two buffer memories are used to sore the LL output of the previous decomposition level. So, for an image size of $512 \times 512 \times 8$ bit the required memory is $4 \times 512=2048$ 8bit memory locations. A and B PE (Process Element) has a $3 \times 3$ working area and $\mathrm{C}$ PE has a 2 x 2 working area.

The computation $\mathrm{s}$ needed for wavelet transform are the above:

$$
\begin{aligned}
& s_{l}^{(0)}=x_{2 l} \quad d_{l}^{(0)}=x_{2 l+1} \\
& d_{l}^{(1)}=d_{l}^{(0)}+a^{*}\left(s_{l}^{(0)}+s_{l+1}^{(0)}\right) \\
& s_{l}^{(1)}=s_{l}^{(0)}+b^{*}\left(d_{l}^{(1)}+d_{l-1}^{(1)}\right)
\end{aligned}
$$

$s_{l}=c^{*} s_{l}^{(1)} \quad d_{l}=c_{-} i n v * d_{l}^{(1)}$

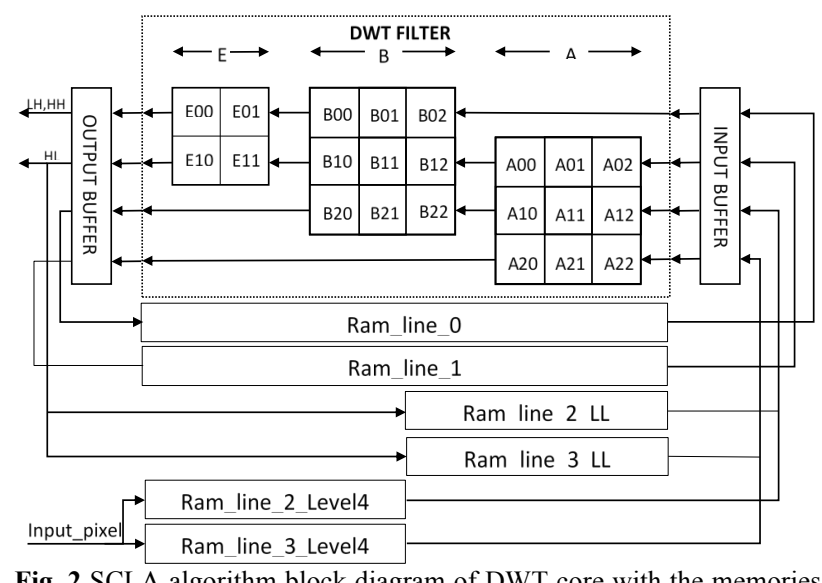

Fig. 2 SCLA algorithm block diagram of DWT core with the memories used.

The wavelet parameters [6] used are:

$$
\begin{aligned}
& a=-\frac{1}{2} \\
& b=\frac{1}{4} \\
& c=\sqrt{2} \\
& c_{i n v}=-\frac{\sqrt{2}}{2}
\end{aligned}
$$

Until now we have described how the SCLA algorithm uses less memory, now we will describe the computation needed to produce the wavelet coefficients.

From figure 2 , it can be seen that there are three processing elements (PE) inside the DWT filter.

Figure 3 shows the PE of both $\mathrm{A}$ and $\mathrm{B}$ elements. The operation that took part is described in as the following. The circles in the four corners means that these elements are as not change. The center pixel, of this $3 \times 3 \mathrm{PE}$, after the operation it will have the same value plus the sums of the four pixels, pointed by the arrows, multiplied by the wavelet parameter. If the $\mathrm{PE}$ is for $\mathrm{A}$ the parameter will be ' $\mathrm{a}$ ' and for B PE will be ' $b$ '. 
The last $\mathrm{PE}$ is $\mathrm{C}$ and it can be seen in the figure 4.In this PE it's easier to compute the result. In the upper right box, the operation made is to multiply the current value with the constant $\mathrm{c}$ and leave it in place. In the downright element is the same operation, the only change is that the constant parameter is $c_{i n v}$.

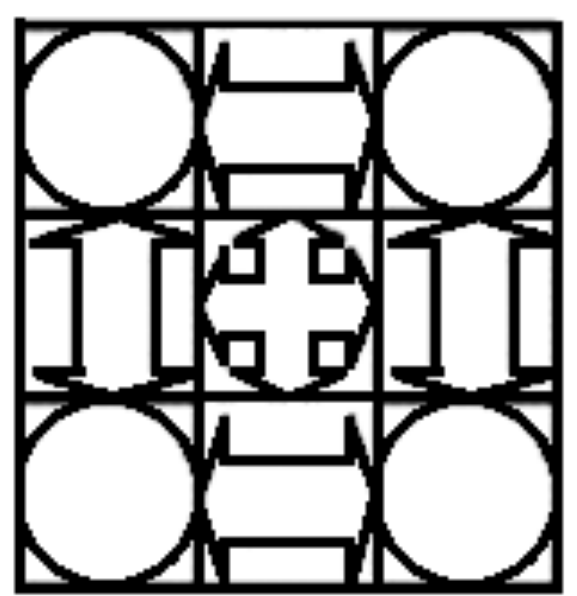

Fig. $3 \mathrm{~A}$ and B PE showing element computations.

In conclusion, it is obvious that with the use of the SCLA algorithm memory and computation reduction can be made. As it expected this algorithm is suitable for systems need to use less memory and reduce the energy consumption like cameras in capsule endoscopy.

By the use of our algorithm we decrease the number of multiplications. In Table 1 you can see a summary.

Table 1 Number of multiplications required by MALLAT, LBI and SCLA algorithm with 5/3 filter.

\begin{tabular}{l|l|c}
\multicolumn{1}{c}{ Algorithm } & \multicolumn{1}{c}{ Level $=1$} & \multicolumn{1}{c}{ Level $=\boldsymbol{J}$} \\
\hline Typical Mallat [8] & $(\mathrm{N} / 2 \times 5+\mathrm{N} / 2 \times 3) \times 2 \mathrm{~N}=8 \mathrm{NxN}$ & $8 / 3 \times\left(4-4^{-\mathrm{j}+1}\right) \times \mathrm{NxN}$ \\
\hline $\begin{array}{l}\text { Symmetrical } \\
\text { Mallat [8] }\end{array}$ & $(\mathrm{N} / 2 \times 3+\mathrm{N} / 2 \times 2) \times 2 \mathrm{~N}=5 \mathrm{~N} \times \mathrm{N}$ & $5 / 3 \times\left(4-4^{-\mathrm{j}+1}\right) \times \mathrm{NxN}$ \\
\hline LBI & $(\mathrm{N} / 2 \times 2+\mathrm{N}) \times 2 \mathrm{~N}=4 \mathrm{NxN}$ & $4 / 3 \times\left(4-4^{-\mathrm{j}+1}\right) \times \mathrm{N} \times \mathrm{N}$ \\
\hline SCLA [7] & $3 / 2 \mathrm{NxN}+\mathrm{N} \times \mathrm{N} / 2=2 \mathrm{NxN}$ & $2 / 3 \times\left(4-4^{-\mathrm{j}+1}\right) \times \mathrm{N} \times \mathrm{N}$ \\
\hline
\end{tabular}

For the implementation and verification of the algorithm we used Xilinx ISE Version 14.3 for implementation and MATLAB R2014a for verification.

By the use of the intergraded tools of Xilinx's software we compose the next table with details of our algorithm in hardware.

Table 2 .Number of resources used by the DWT algorithm.

\begin{tabular}{c|c|c|c}
\hline & $\begin{array}{c}\text { Nr. Of Slice } \\
\text { Registers }\end{array}$ & Nr. OfLUTs & $\begin{array}{c}\text { Nr. Of LUT-FF } \\
\text { pairs }\end{array}$ \\
\hline DWT_core & 759 & 1077 & 417 \\
\hline Memory & 24576 & 42642 & 24576 \\
\hline Total: & 25335 & 43719 & 24993 \\
\hline
\end{tabular}

\subsection{Wireless transmission}

The ability of the capsule to achieve high quality diagnostic results mostly relies on the wireless communication link and its characteristics. Endoscopic capsules have some characteristics which are unchangeable, like its size. It is obvious that with this small size the energy that can be stored, is too low. So, the power consumption is a big criterion for the design of the whole system and especially of the wireless link.

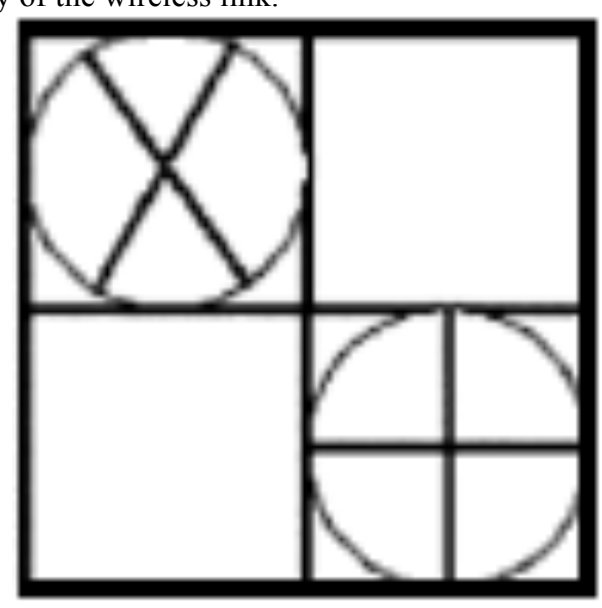

Fig. $4 \mathrm{C}$ PE element computations

The transition of compressed image data needs highdata-rate modulation schemes. Preferred schemes for such applications are QPSK (quadrature phase shift keying), FSK and ASK. For this type of medical application due to its low complexity FSK modulation is preferred. A unidirectional wireless link is proposed in paper [9]. But it is still a high energy hungry scheme with complex building blocks such as PLL (Phase-Locked loop) and analogue to digital converters. In this paper we select to design a unidirectional wireless link based on the OOK (On-OFF keying) modulation which belongs to the ASK family. With a few external components we can design the OOK transmitter. The modulation is made inside the FPGA and by the use of one pin we transmit the data. Taking advantage of FPGA's technology, we use the differential input pins LVDS (Low-voltage differential signalling) to implement the OOK receiver. The demodulation is taking place inside the FPGA. This work [10] suggests using a carrier between 450 and $900 \mathrm{MHz}$ for maximum radiation. But from experiments [11] is showed that the smaller the carrier frequency there is less interference in the signal from the surrounding tissues and higher signal-to-noise ratio (SNR). The selection of the carrier frequency is based on the above. Furthermore, a low frequency uses less energy to be produced. From all these the selection of the carrier frequency is in the $2 \mathrm{~m}$ amateur band which is between $144 \mathrm{MHz}$ to $148 \mathrm{MHz}$. For antenna system the idea used is based on the regions of electromagnetic field. There is the near field and the far field. The far field is the region that works as normal electromagnetic radiation. The main difference that we take advantage is that the near field strength decreases dependent on the distance from the antenna whereas in far field the strength decreases by the square of the distance. In magnetic RFID systems both transmitter and receiver are working in the near field region. So, the use inductive couples coils can achieve the desired results. Antenna

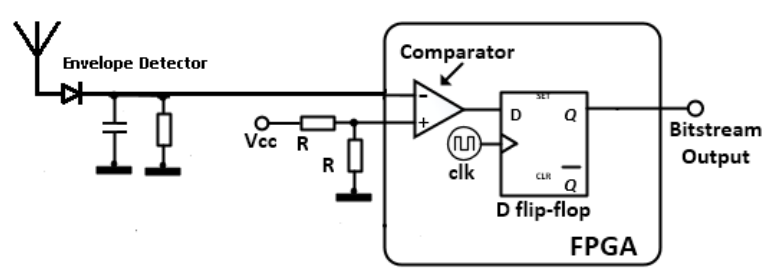

Fig. 5 Wireless receiver and demodulator implementation. 


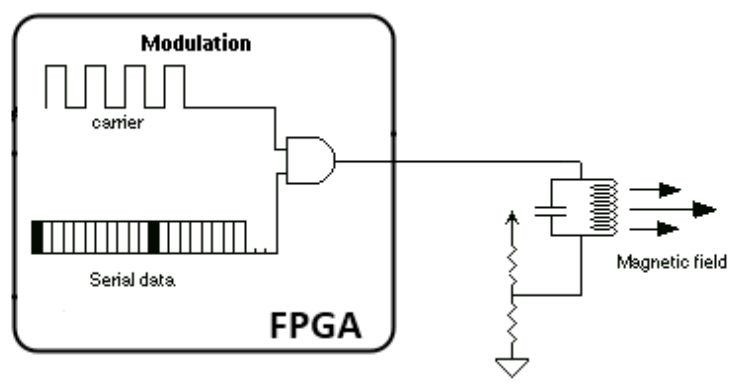

Fig. 6 Wireless transmitter and modulator implementation.

Figure 5 shows our implementation of the wireless receiver with the external components used and Figure 6 shows the transmitter.

\section{Conclusions}

In this paper, a new design of a wireless swallowable pill has been proposed. We propose a novel algorithm for 2-D Discrete Wavelet Transform implemented with 5/3 tap filter, based on lifting scheme, managing to introduce a lossless compression identical for medical applications. The DWT processor can handle both row and columns data the same time due to its architecture. It uses just 3 DSP modules of FPGA's resources and has lower power consumption, less memory usage and hardware utilization.

The wireless system implemented in the FPGA and the exceptional features of it let us design a low power, less external components, high data rate unipolar wireless system.

In addition, Catadioptric omnidirectional lenses are proposed to use in this system. They all together let our system capture high resolution images with low energy consumption.

This paper was presented at Pan-Hellenic Conference on Electronics and Telecommunications - PACET, that took place May 8-9 2015, at Ioannina Greece.

\section{References}

1. Given Imaging Ltd. [Online]. http://www.givenimaging.com/en-int/Innovative-

Solutions/Capsule-Endoscopy/Pillcam-ESO/Pages/default.aspx [Accessed 24 March 2015].

2. McKenzie, J. and Osgood, D. Validation of a new telemetric core temperature monitor. J. Therm. Biol., 2004, 29, 605-611.

3. Gonzalez-Guillaumin, J., Sadowski, D., Kaler, K., and Mintchev, M. "Ingestible capsule for impedance and $\mathrm{pH}$ monitoring in the esophagus." IEEE Trans. Biomed. Engng, 2007, 54, 2231-2236.

4. Shree K. Nayar. "Catadioptric Omnidirectional Camera" IEEE, 2007, pp. 482-488.

5. Silica[Online]. http://www.silica.com/product/spartan-6-fpgaindustrial-video-processing-kit.html [Accessed 26 March 2015].

6. Leibo Liu, Ning Chen, Hongying Meng, Li Zhang, Zhihua Wang, and Hongyi Chen. "A VLSI Architecture of JPEG2000 Encoder" IEEE journal of Solid-State Circuits, Vol. 39, no. 11, November 2004, pp 2032-2040.

7. H. Meng and Z. Wang, "Fast spatial combinative lifting algorithm of wavelet transform using the $9 / 7$ filter for image block compression," Electron. Lett., vol. 36, no. 21, Oct. 12, 2000, pp. 1766-1767.

8. MALLAT, S.: "A theory for multiresolution signal decomposition: the wavelet representation", IEEE Trans. Patt. Anal. Mach. Intell.,1989, 11, pp. 674-693.

9. J. Thonı, S. Radiom, D. Turgis, R. Carta, G. Gielen, R. Puers, "Design of a 2 Mbps FSK near-field transmitter for wireless capsule endoscopy". Sensors and Actuators A 156 (2009) 43-48.

10. L.C. Chirwa, P.A. Hammond, S. Roy, D.R.S. Cumming, Electromagnetic radiation from ingested sources in the human intestine between $150 \mathrm{MHz}$ and $1.2 \mathrm{GHz}$, IEEE Trans. Biomed. Eng. 50 (4) (2003) 484-492.

11. L.Wang, T.D. Drysdale, D.R.S. Cumming, In-situ characterization of two wireless transmission schemes for ingestible capsules, IEEE Trans. Biomed. Eng. 54 (11) (2007) 2020-2027.

12. I. Daubechies and W. Sweldens, "Factoring wavelet transforms into lifting steps," J. Fourier Appl., vol. 4, pp. 247-269, 1998.
13. Stephane G. Mallat, "A Theory for Multiresolution Signal Decomposition:The Wavelet Representation", IEEE Transactions on Pattern Analysis and Machine Intelligence, vol. 11, no. 7, Jul.1989, pp. 674-693.

14. Marc Antonini, Michael Barlaud, Pierre Mathieu and Ingrid Daubechies, "Image Coding Using Wavelet Transform", IEEE Transactions on Image Processing, vol. 1, no. 2, Apr. 1992, pp. 205-220.

15. Maria E. Angelopoulou, Peter Y.K. Cheung, Konstantinos Masselos and Yiannis Andreopoulous"Implementation and Comparison of the 5/3 Lifting 2D Discrete Wavelet Transform Computation Schedules on FPGAs", Journal of Signal Processing Systems, Springer, vol. 51, 2008, pp.3-21.

16. Liu Hong-jin, Shao Yang, He Xing, Zhang Tie-jun, Wang Donghui and Hou Chao-huan, "A Novel VLSI Architecture for 2-D Discrete Wavelet Transform", IEEE International Conference on ASIC, Oct. 2007, pp. 40-43.

17. Xin Tian, Lin Wu, Yi-Hua Tan, and Jin-Wen Tian, "Efficient Multi- Input/Multi-Output VLSI Architecture for TwoDimensional Lifting- Based Discrete Wavelet Transform", IEEE Transactions on Computers, vol. 60, no. 8, Aug. 2011, pp. 12071211.

18. C. Chrysafis and A. Ortega, "Line based, reduced memory, wavelet image compression," IEEE Trans. Image Processing, vol. 9, Mar. 2000, pp. 378-389.

19. Sang Won Seo, Seungoh Han, Jun Ho Seo, Woo Bum Choi and Man Young Sung, "Liquid Lens Module with Wide Field-ofView and Variable Focal Length". Electronic Materials Letters, Vol. 6, No. 4 (2010), pp. 141-144.

20. Carta, R., "Wireless power and data transmission for robotic capsule endoscopes". Communications and Vehicular Technology in the Benelux (SCVT), 2011 18th IEEE Symposium on 2011.

21. Moglia, A., Menciassi, A., Dario, P., and Cuschieri, A. "Capsule endoscopy: progress update and challenges ahead." Nat. Rev. Gastroenterol. Hepatol., 2009, 6, 353-362. 